\title{
Absent filling of the superficial middle cerebral vein is associated with reperfusion but not parenchymal hematoma in stroke patients undergoing thrombectomy: an observational study
}

\author{
Sheng Zhang ${ }^{1 \#}$, Ruiting Zhang ${ }^{2 \#}$, Bo Jin ${ }^{1}$, Zongjie Shi ${ }^{1}$, Chenguang Li $^{3}$, Yannan Yu ${ }^{4}$ Zhen Wang ${ }^{5}$ \\ ${ }^{1}$ Department of Neurology, People's Hospital of Hangzhou Medical College, Zhejiang Provincial People's Hospital, Hangzhou, China; ${ }^{2}$ Department \\ of Radiology, ${ }^{3}$ Department of Neurosurgery, The Second Affiliated Hospital of Zhejiang University, School of Medicine, Hangzhou, China; \\ ${ }^{4}$ Department of Radiology, School of Medicine, Stanford University, Los Angeles, USA; ${ }^{5}$ Department of Radiology, People's Hospital of Hangzhou \\ Medical College, Zhejiang Provincial People's Hospital, Hangzhou, China \\ Contributions: (I) Conception and design: S Zhang, R Zhang, Z Wang; (II) Administrative support: S Zhang, Z Wang; (III) Provision of study \\ materials or patients: S Zhang, B Jin, Z Shi; (IV) Collection and assembly of data: S Zhang, B Jin; (V) Data analysis and interpretation: S Zhang, R \\ Zhang, C Li, Z Wang; (VI) Manuscript writing: All authors; (VII) Final approval of manuscript: All authors. \\ \#These authors contributed equally to this work. \\ Correspondence to: Zhen Wang, MBBS. Department of Radiology, People's Hospital of Hangzhou Medical College, Zhejiang Provincial People's \\ Hospital, 158\# Shangtang Road, Hangzhou 310014, China. Email: wang12345zhen@sina.com.
}

Background: Parenchymal hematoma $(\mathrm{PH})$ is the most feared complication of reperfusion therapy after stroke. The opacification of the superficial middle cerebral vein (SMCV) on computed tomography perfusion (CTP) has been associated with poor functional outcomes after stroke, while its association with PH has not been verified for acute stroke patients undergoing thrombectomy.

Methods: Consecutive patients with acute anterior large artery occlusion (LAO) who received thrombectomy were retrospectively enrolled between May 2018 and May 2019. Absent filing of the SMCV (SMCV-) on CTP-derived CT angiography was defined as no contrast filling of the SMCV across the whole venous phase in the ischemic hemisphere, while $\mathrm{SMCV}+$ was defined as the presence of contrast filling of the SMCV at any time point of the venous phase.

Results: A total of 52 patients were enrolled in the study, and 15 patients (28.8\%) developed a $\mathrm{PH}$ within 48 hours after thrombectomy. SMCV- was not associated with $\mathrm{PH}$ in both the univariate and multivariate logistic regression analyses (all $\mathrm{P}>0.05$ ), but was an independent risk factor for reperfusion [modified thrombolysis in cerebral infarction score of $2 \mathrm{~b}-3$; odds ratio $(\mathrm{OR})=0.172,95 \%$ confidence interval (CI): 0.031-0.960, $\mathrm{P}=0.045]$. Reperfusion was associated with a reduced risk of $\mathrm{PH}$ (OR $=0.110,95 \%$ CI: $0.013-$ $0.913, \mathrm{P}=0.041)$. However, in a subgroup analysis of patients who had reperfusion, the SMCV-group had a higher rate of $\mathrm{PH}$ than the $\mathrm{SMCV}+$ group $(40.0 \%$ vs. $13.8 \%, \mathrm{P}=0.049)$.

Conclusions: In patients who received thrombectomy, SMCV- did not predict $\mathrm{PH}$, but was a risk factor for reperfusion. Although reperfusion was a protective factor for $\mathrm{PH}$, the SMCV-group was still at a higher risk of $\mathrm{PH}$ compared with the SMCV+ group when reperfusion was successfully achieved.

Keywords: Superficial middle cerebral vein (SMCV); parenchymal hematoma (PH); thrombectomy; reperfusion

Submitted Feb 01, 2020. Accepted for publication Aug 21, 2020.

doi: 10.21037/atm-20-1154

View this article at: http://dx.doi.org/10.21037/atm-20-1154 


\section{Introduction}

Hemorrhagic transformation (HT) is the most feared complication of reperfusion therapy after acute ischemic stroke. HT is classified into two major categories based on the European Cooperative Acute Stroke Study (ECASS) definition: hemorrhagic infarct (HI) and parenchymal hematoma (PH) (1). The latter type has been associated with poor outcomes in ischemic stroke patients, especially for those who received intra-arterial therapy $(2,3)$. Thus, the detection of $\mathrm{PH}$ may be a useful tool to guide the management of acute stroke.

Recently, the cerebral venous system was found to play a conflicting role in the prediction of $\mathrm{PH}(4,5)$. The superficial middle cerebral vein (SMCV) is a large superficial cerebral vein which can be easily identified on imaging. We have previously found that the absent filling of the SMCV (referred to as $\mathrm{SMCV}-$ ) was associated with brain edema expansion and poor functional outcomes in acute ischemic stroke, but was not associated with PH (6). We considered that the SMCV- on the ischemic side represented venous blood stagnation or even thrombosis within cerebral venules of the SMCV drainage area. Therefore, SMCVpatients were unlikely to suffer a $\mathrm{PH}$ because of a lack of cerebral blood perfusion within the ischemic area if arterial clots were not removed. However, the majority of enrolled patients had no large cerebral artery occlusions (LAO) and did not receive thrombectomy.

It has also been reported that abnormal venous outflow may hinder the reperfusion of ischemic brain tissue in LAO even after successful recanalization (7). At present, there is a lack of research clarifying the relationship between SMCV and $\mathrm{PH}$ after cerebral blood perfusion restoration through thrombectomy in patients with acute LAO. Therefore, in this study, we aimed to investigate the relationship between SMCV-, reperfusion, and $\mathrm{PH}$ in acute LAO patients.

We present the following article in accordance with the STROBE reporting checklist (available at http://dx.doi. org/10.21037/atm-20-1154).

\section{Methods}

\section{Ethics statement}

The study was approved by the institutional ethics committee (NO. KY2017019), and informed consent was obtained from all patients. All clinical investigations were conducted according to the principles expressed in the Declaration of Helsinki (as revised in 2013).

\section{Setting}

We retrospectively reviewed our prospectively collected database for consecutive acute LAO patients who received computed tomography perfusion (CTP) scan at admission and thrombectomy within 6 hours after stroke onset from May 2018 to May 2019 in Zhejiang Provincial People's Hospital.

\section{Participants}

Patients were included if they had (I) a middle cerebral artery (MCA) segment 1 (M1) and/or intracranial internal carotid artery (ICA) occlusion on pre-operative 4D computed tomography angiography (4D-CTA) scans reconstructed from CTP; (II) a pre-stroke modified Rankin Scale (mRS) score $\leq 2$; (III) complete imaging and clinical data during hospitalization. Patients were excluded if they had bilateral acute ischemic lesions or poor image quality due to motion artifacts (see Figure 1).

\section{Reperfusion therapy}

Patients who arrived at our center within 4.5 hours of symptom onset with no hemorrhage or significant lowdensity lesions on baseline non-contrast head CT (NCCT) images received alteplase at a dose of $0.9 \mathrm{mg} / \mathrm{kg}$ (maximum dose $=90 \mathrm{mg}$ ). However, failure to receive thrombolysis did not preclude patients from thrombectomy. Patients with no clinical improvement after thrombolysis and patients who arrived 4.5-6 hours after stroke onset were thrombectomy candidates. According to the 2018 American Heart Association/American Stroke Association (AHA/ASA) guidelines, candidates were selected for thrombectomy if they met all of the following criteria (8): (I) pre-stroke mRS score of $0-1$; (II) a causative occlusion of the ICA or MCA M1 segment; (III) aged $\geq 18$ years; (IV) National Institutes of Health stroke scale (NIHSS) score of $\geq 6$; (V) Alberta stroke program early CT score (ASPECTS) of $\geq 6$; and (VI) treatment can be initiated (groin puncture) within 6 hours of symptom onset. Patients with any contraindication for thrombolysis who were eligible for thrombectomy also received thrombectomy directly.

Thrombectomy was performed as a bridging therapy after intravenous thrombolysis, or as primary therapy following the current guidelines. All patients who received thrombolysis or thrombectomy provided informed consent. The retrieval device for thrombectomy was the 




Figure 1 Study flowchart. LAO, large artery occlusion; CTP, computed tomography perfusion; mRS, modified Rankin Scale.

Solitaire AB (ev3, Covidian, Dublin, Ireland). The details of the surgical procedure performed are described in the EXTEND-IA trial (9). As the auxiliary treatment of thrombectomy, the use of tirofiban was initiated during and after thrombectomy. Patients received an initial dose of $0.4 \mathrm{mg}$ over 30 minutes, and tirofiban administration was then continued at a dosage of $0.1 \mathrm{mg} / \mathrm{kg}$ body weight/hour. The recommended infusion duration was 24 hours.

\section{Imaging protocols}

All patients underwent baseline NCCT and CTP, and followup NCCT at 24-48 hours after admission. All CT imaging was acquired on a 320-detector row 640-slice cone-beam multidetector CT (MDCT) scanner (Aquilion One, Toshiba Medical Systems). Whole-brain NCCT was performed in one rotation (detector width $16 \mathrm{~cm}$ ). After NCCT, a CTP was acquired after administration of $50 \mathrm{~mL}$ of contrast agent (Ultravist 370; Bayer HealthCare, Berlin, Germany) injected intravenously at a rate of $6 \mathrm{~mL} / \mathrm{second}$ chased by $50 \mathrm{~mL}$ of saline (acquisition parameters: $120 \mathrm{kV}, 128 \mathrm{mAs}$, scanning coverage $=240 \mathrm{~mm}$, scanning width $=5 \mathrm{~mm}$ ). Starting 7 seconds after contrast injection, a pulsed full rotation scan with 18 -time points acquired over 60 seconds with a total pulse image acquisition time of 9.5 seconds was performed. The scanning protocol of NCCT performed 24 hours postprocedure was the same as that of baseline NCCT.

\section{Imaging analysis}

Two raters (S Zhang and Z Wang) who jointly evaluated the imaging characteristics were blinded to the patients' followup imaging and clinical data. A single trained observer ( $\mathrm{S}$ Zhang) measured imaging markers in all patients twice, at an interval of 1 month apart. Another observer (Z Wang) independently made the same evaluation.

\section{Assessment of arterial collaterals}

Multi-phase CTA, including arterial peak phase, venous peak phase, and late venous phase images, were generated according to the arterial input function/venous output function (AIF/VOF) curves by Vitoria ${ }^{\circledR} \mathrm{fX}$ software (Version 1.0, Vital Images, Minnetonka, MN, USA). Leptomeningeal collaterals were assessed on multi-phase CTA using the method previously described in the ESCAPE trial (10). We divided patients into two groups: good and intermediate collaterals group, and poor collaterals group.

\section{Absent filling of ipsilateral SMCV (SMCV-)}

We assessed the SMCV on 4D-CTA reconstructed from CTP using Vitrea ${ }^{\circledR}$ fX (Version 1.0, Vital Images, Minnetonka, MN, USA). Images were analyzed using maximum intensity projection (MIP). SMCV was defined as negative (SMCV-) if no contrast filling of the SMCV across the whole venous phase in the ischemic hemisphere was present. The presence of contrast filling in the ipsilateral SMCV at any time point of the venous phase was classified as SMCV+ (refer to Figure 2). Patients with contralateral absent contrast filling of the SMCV only were also ascribed to the $\mathrm{SMCV}+$ group. 


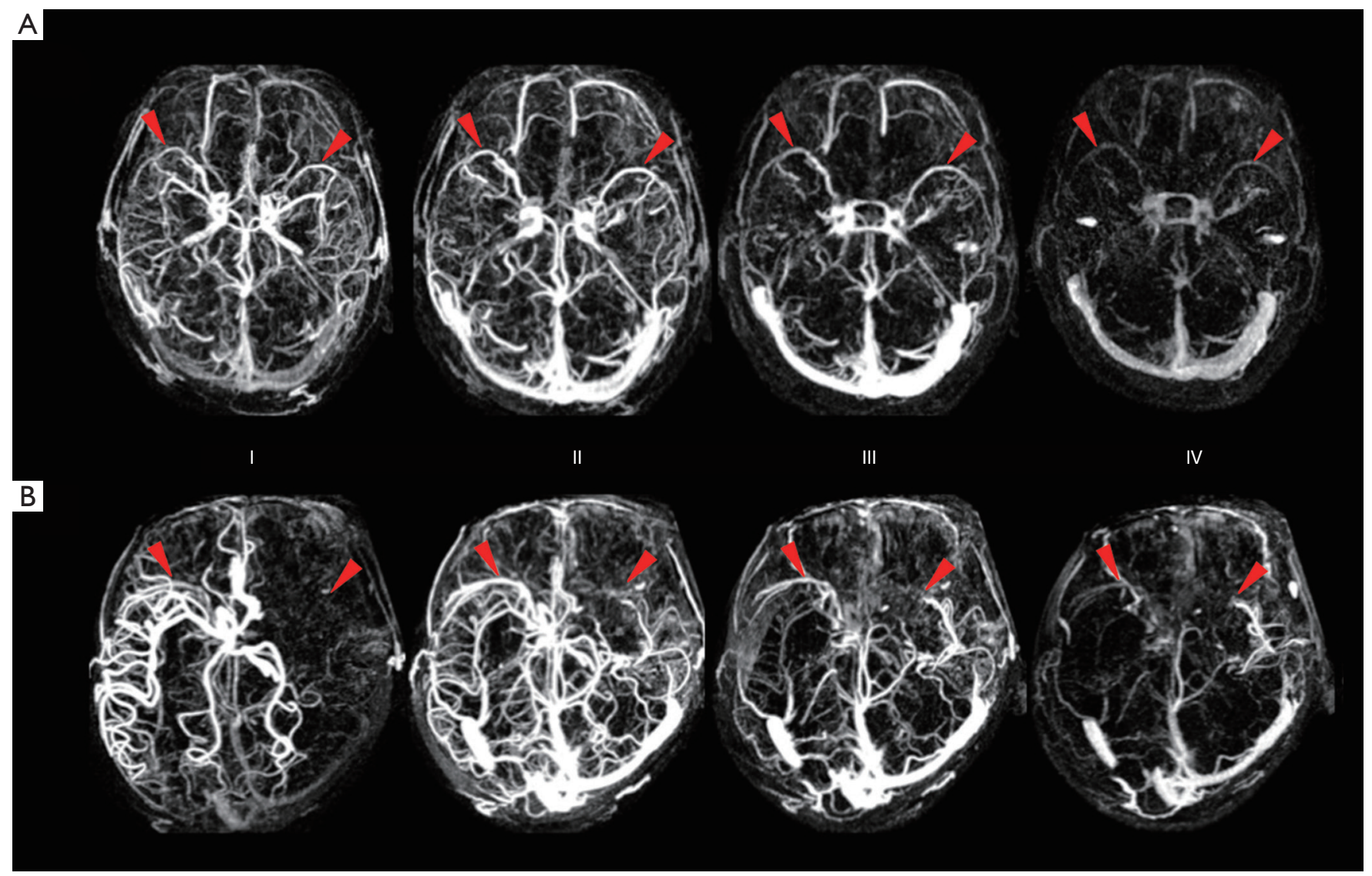

Figure 2 Examples of opacification of the SMCV on dCTA in healthy and acute LAO patients. It is known that cerebral veins are opacified in sequences. As surface cortical veins are opacified early in the venous phase, SMCV can be seen in this phase (I). The mid-venous phase (II and III) is always the best phase to view the overall picture of the cerebral veins as more downstream venous channels can be visualized. While in the late venous phase (IV), the contrast within the SMCV can be almost cleared out as most superficial veins are no longer visualized in this phase. Bilateral symmetrical SMCV opacification (red triangular arrow) can be seen in the healthy control subject from the early to late phase (see patient A, I-IV). According to this regularity, the SMCV was defined as negative (SMCV-) if no contrast filling of the SMCV across the whole venous phase in the ischemic hemisphere was found; otherwise, it was defined as SMCV+. In patient B who had an acute left intracarotid artery occlusion, the ipsilateral SMCV (see the left red triangular arrow) that was not present on dCTA from the early to late venous phase was marked as SMCV-. SMCV, superficial middle cerebral vein; dCTA, dynamically computed tomography angiography; LAO, large artery occlusion.

\section{Neurological outcome measures}

NIHSS scores were evaluated and recorded at baseline, 24-72 hours after admission (as NIHSS within 24 hours was not acquired in some patients who received oral intubations or general anesthesia for thrombectomy), and at any time if a patient's neurological status worsened.

Reperfusion and recanalization status were both assessed on digital subtraction angiograms (DSA) using modified thrombolysis in cerebral infarction (mTICI) scores (11) and arterial occlusive lesions (AOL) (12) respectively in patients who received thrombectomy. The mTICI and AOL scores were assigned following completion of the thrombectomy procedure. We defined an mTICI score of $0-2 \mathrm{a}$ as no reperfusion and a score of $2 \mathrm{~b}-3$ as reperfusion. AOL scores of $0-1$ were defined as no recanalization, and scores of 2-3 were classified as recanalization.

HT was classified as HI or PH, according to the ECASS definition (1). Midline shift was defined as the displacement of the septum pellucidum (or cavum septi pellucidi) more than $3 \mathrm{~mm}$ from the spatial midline. HT 
and midline shift (13) were both assessed on the $24-$ 48-hour NCCT scans.

mRS scores were used to identify the clinical outcome at discharge. mRS scores of $0-3$ were classified as a good outcome, and mRS scores of 4-6 were classified as a poor outcome.

\section{Statistical analysis}

Cohen's kappa coefficient was used to assess the level of inter- and intra-observer agreement for detecting the presence of SMCV-, poor collaterals, midline shift, and $\mathrm{PH}$. Excellent inter- and intra-observer agreement was seen in distinguishing the SMCV- $(\kappa=0.918$ and 0.879$)$, poor collaterals $(\kappa=0.912$ and 0.824$)$, midline shift $(\kappa=0.906$ and $0.803)$ and $\mathrm{PH}(\kappa=0.924$ and 0.954$)$.

All numeric variables were expressed as mean \pm standard deviation (SD) and median (interquartile range, IQR). Categorical variables were presented as frequency (percentage). Fisher's exact test was used to compare dichotomous variables between groups, while the MannWhitney $U$ test was used for ordered categorical variables. An independent-samples two-tailed $t$-test or Mann-Whitney $U$ test was used for continuous variables, depending on the normality of the distribution. Variables identified by univariate analysis $(\mathrm{P}<0.1)$ were included in the binary logistic regression model. All analyses were performed with blinding of the participants' identifying information. Statistical analysis was performed using SPSS 18 (SPSS Inc., Chicago, IL, USA).

\section{Results}

\section{Patient characteristics}

A total of 52 patients were enrolled in the study (Figure 1). Patients had a median age of 71.5 years (IQR, 61.3-79 years), and 15 (28.8\%) were females. The median baseline NIHSS score was 20 (IQR, 16-23). SMCV- was found in 21 patients (40.4\%) (univariate comparisons between SMCV- and SMCV+ are shown in Table S1). A total of 18 patients $(34.6 \%)$ underwent bridging therapy, and $34(65.4 \%)$ underwent thrombectomy alone. After thrombectomy, 44 patients had successful reperfusion (84.6\%), and 15 (28.8\%) had PH within 48 hours after thrombectomy. In patients who suffered $\mathrm{PH}, 60 \%$ had a midline shift, and $80 \%$ had poor outcomes. Baseline and post-thrombectomy clinical and radiological characteristics of all 52 patients are described in Table 1.

\section{The association between $\mathrm{PH}$ and SMCV-}

Before thrombectomy, compared with the non-PH group $(n=37)$, the PH group $(n=15)$ had a higher rate of poor collaterals and a lower rate of intravenous thrombolysis. The presence of SMCV- was not significantly different between the non-PH and $\mathrm{PH}$ group (Table 1). We enrolled in the SMCV- group, poor collaterals group, and the intravenous thrombolysis group into a binary logistic regression analysis and found that only intravenous thrombolysis was independently associated with $\mathrm{PH}$ [odds ratio $(\mathrm{OR})=0.176,95 \%$ confidence interval $(\mathrm{CI}): 0.031-$ 0.996, $\mathrm{P}=0.049]$. After thrombectomy, patients with $\mathrm{PH}$ had a lower rate of reperfusion, a higher 24-hour follow-up NIHSS score, a higher rate of midline shift, decompressive craniectomy, and poor outcomes at discharge (Table 1). We further added reperfusion into the binary logistic regression analysis and found that intravenous thrombolysis (OR $=0.104,95 \%$ CI: $0.013-0.831, \mathrm{P}=0.033)$ and reperfusion (OR $=0.110,95 \%$ CI: 0.013-0.913, $\mathrm{P}=0.041)$ were both independently associated with $\mathrm{PH}$, while $\mathrm{SMCV}$ - was still not associated with $\mathrm{PH}(\mathrm{OR}=0.722$, 95\% CI: 0.102-5.090, $\mathrm{P}=0.744)$ (see Table S2).

\section{The relationship between $S M C V$-, reperfusion, and $\mathrm{PH}$}

There was no significant difference in the rate of SMCVbetween the recanalization and no recanalization group ( $37.8 \%$ vs. $57.1 \%, \chi^{2}=0.944, \mathrm{P}=0.331$ ). A significantly lower rate of SMCV- was found in the reperfusion group compared with the no reperfusion group $(34.1 \%$ vs. $75.0 \%$, $\chi^{2}=4.705, \mathrm{P}=0.030$ ) (Table S3). Binary logistic regression analysis showed that $\mathrm{SMCV}$ - was an independent risk factor for reperfusion $(\mathrm{OR}=0.172,95 \% \mathrm{CI}: 0.031-0.960$, $\mathrm{P}=0.045$ ).

In the reperfusion group, patients with SMCV- had a higher rate of $\mathrm{PH}$ than patients with $\mathrm{SMCV}+(40.0 \%$ vs. $13.8 \%, \chi^{2}=3.866, \mathrm{P}=0.049$ ) (see Table 2). In patients with $\mathrm{SMCV}-$, the rates of $\mathrm{PH}$ were similar between the no reperfusion and reperfusion group (50\% vs. $40.0 \%$, $\left.\chi^{2}=0.175, \mathrm{P}=0.676\right)$. In patients with $\mathrm{SMCV}+$, the rate of $\mathrm{PH}$ was significantly lower in the reperfusion group compared to the no reperfusion group $(13.8 \%$ vs. $\left.100 \%, \chi^{2}=8.908, P=0.032\right)$. Examples of the relationship between $\mathrm{SMCV}-$, reperfusion, and $\mathrm{PH}$ are shown in Figure 3. 
Table 1 Baseline and post-thrombectomy clinical and imaging characteristics of patients stratified by non-PH vs. $\mathrm{PH}$

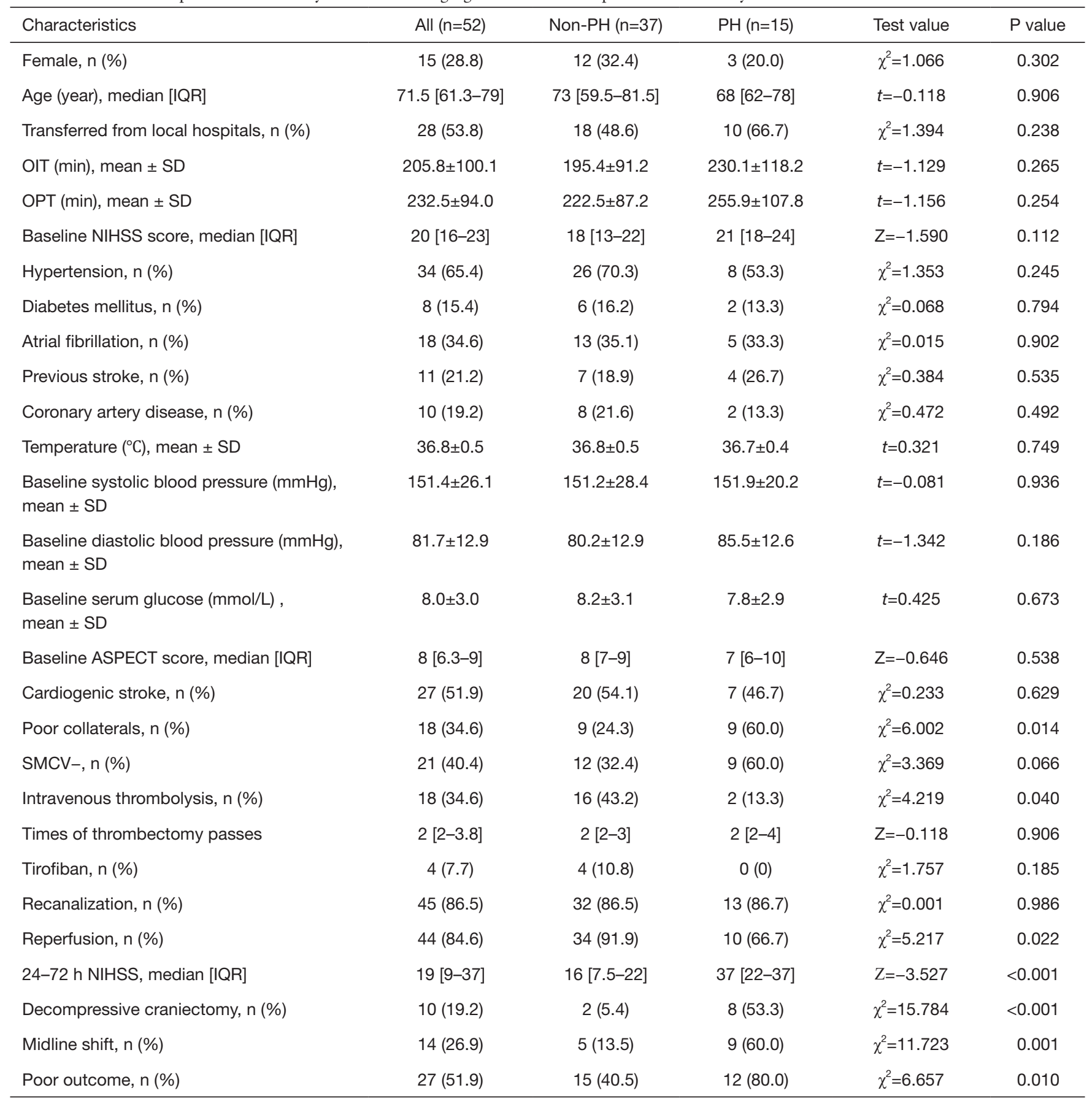

ASPECT, Alberta stroke program early CT score; PH, parenchymal hematoma; SD, standard deviation; IQR, interquartile range; OIT, onset to imaging time; OPT, onset to puncture time; NIHSS, National Institute of Health stroke scale; SMCV-, absent filling of the ipsilateral superficial middle cerebral vein. 
Table 2 Comparison of the outcomes in patients who achieved successful reperfusion through thrombectomy between SMCV-and SMCV+ subgroups

\begin{tabular}{|c|c|c|c|c|}
\hline Outcomes & SMCV+ & SMCV- & Test value & $P$ value \\
\hline Midline shift, n (\%) & $1(3.4)$ & $10(66.7)$ & 21.073 & $<0.001$ \\
\hline Decompressive craniectomy, n (\%) & $0(0)$ & $7(46.7)$ & 16.094 & $<0.001$ \\
\hline Poor outcome, n (\%) & $8(27.6)$ & $13(86.7)$ & 13.832 & $<0.001$ \\
\hline
\end{tabular}

$\mathrm{PH}$, parenchymal hematoma; SMCV, superficial middle cerebral vein.

\section{Discussion}

In this study, we found that SMCV- was a risk factor for reperfusion, but not for $\mathrm{PH}$. Furthermore, in patients who achieved successful reperfusion, SMCV- had a higher rate of $\mathrm{PH}$ than $\mathrm{SMCV}+$. The occurrence of $\mathrm{PH}$ can be influenced by intravenous thrombolysis before thrombectomy and reperfusion through thrombectomy.

In patients who received thrombectomy, the presence of SMCV - did not interfere with the rate of recanalization, meaning that the abnormality of SMCV drainage has no impact on recanalization since this mainly depends on the stent device or the characteristics of the clots. However, we found that SMCV- patients were unlikely to achieve successful reperfusion after thrombectomy. This result indicated that SMCV - at baseline might be involved in the hindrance of peripheral reperfusion even after arterial occlusion was resolved. This finding is consistent with the "no-reflow" phenomenon described by Gerber et al., where although the arterial opacification is normal, there is still a lack of contrast flow to the venous side after endovascular therapy (7). The occurrence of this phenomenon is most likely associated with "cerebral venous steal". After a stroke, ischemia may damage small veins and venules, causing blood clot formation in the venous system. In this case, severe ischemia may lead to little outflow pass through the venous side, and the restored arterial blood flow by successful recanalization will diverge to the penumbra rather than to the core (the most ischemic area), thus "noreflow" emerges $(14,15)$.

In this study, reperfusion was found to be protective for the occurrence of $\mathrm{PH}$, while SMCV- patients had a higher rate of $\mathrm{PH}$ than $\mathrm{SMCV}+$ patients after reperfusion was successfully achieved (40\% vs. 13.8\%). Even after successful reperfusion from thrombectomy, the occurrence of $\mathrm{PH}$ was still high in SMCV- patients with the recovery of tissue pressure. Two mechanisms possibly explain this phenomenon. Firstly, the arterioles dilate 5-10 times the original lumen size because of the release of adenosine and nitric oxide from dying cells and the endothelium after brain ischemia (16). Zhang et al. also found that during cerebral ischemia, the arterial smooth muscle in downstream arteries from the clots may lose contractile ability to adjust blood flow in line with heart rate and blood pressure (17). In this case, when accompanied by drainage abnormalities of cerebral veins, we speculated that more blood might enter into the brain parenchyma resulting in reperfusion injury if the arterial clots were being removed by a stent retriever or a recombinant tissue plasminogen activator (rtPA). Secondly, venules were also found to modulate the autoregulation of cerebral blood flow. Venules can have a protective effect by constricting pial veins to prevent the abrupt increase in venous pressure (18). However, in the acute phase after stroke, this protective effect could fail. When reperfusion happens, capillary and venules suffer a sudden elevation of perfusion pressure leading to regional hyperemia and disturbance of the venous brainblood-barrier $(19,20)$. Although it is the venule side that is reported to suffer HT due to brain-blood-barrier abruption and increased perfusion, it is not possible to evaluate the bleeding site in our population, which requires advanced imaging techniques in future studies.

Notably, we found that patients who received bridging therapy had a significantly lower rate of PH. The outcomes of direct thrombectomy vs. bridging therapy for acute LAO patients have long been debated. Maingard et al. reported that patients who received bridging therapy had a higher rate of reperfusion and functional independence (mRS $0-2$ at 90 days) (21). Contrastingly, a network meta-analysis of 12 studies with a total of 3,161 patients showed that there was no significant difference in good functional outcome at 90 days between direct thrombectomy and bridging therapy (22). However, a comparison of the occurrence of $\mathrm{PH}$ between 


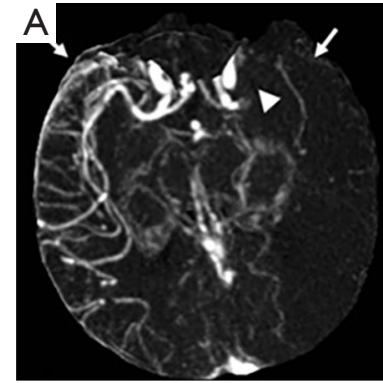

Baseline 4D-CTA

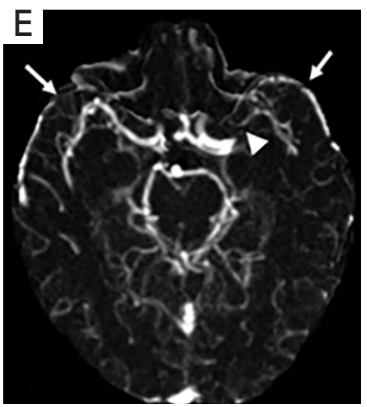

Baseline 4D-CTA



Final angiography after thrombectomy

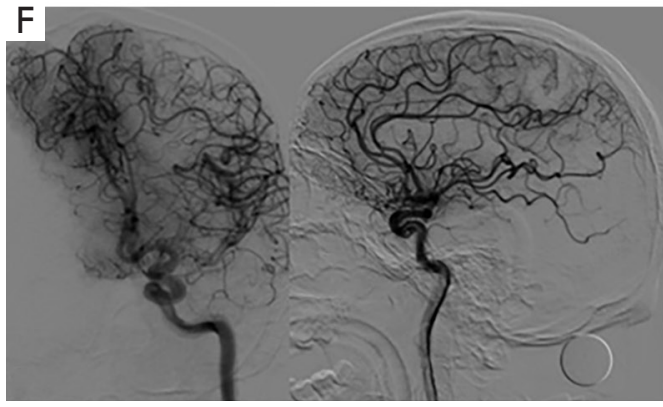

Final angiography after thrombectomy



$6 \mathrm{~h}$ NCCT after thrombectomy

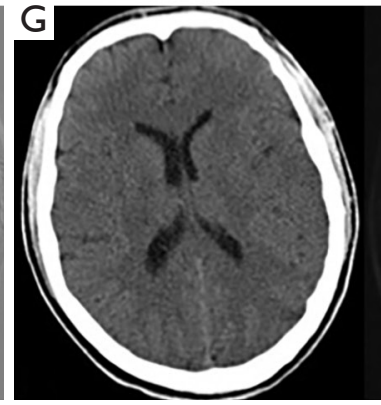

$24 \mathrm{~h} \mathrm{NCCT} \mathrm{after} \mathrm{thrombectomy}$

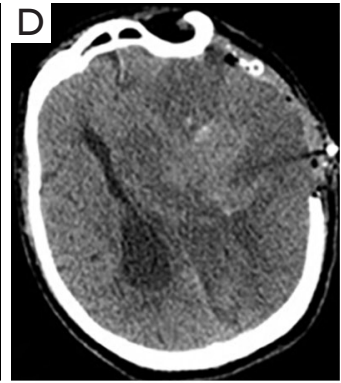

$24 \mathrm{~h} \mathrm{NCCT}$ after thrombectomy

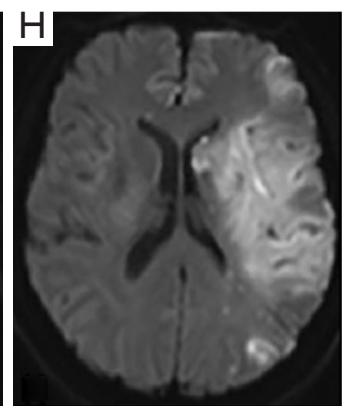

$72 \mathrm{~h}$ DWI after thrombectomy

Figure 3 The association between contrast filling of the SMCV with reperfusion and PH. Patient 1: A 72-year-old male with an acute left middle cerebral artery occlusion (MCAO; baseline NIHSS $=21$ ) (white triangle arrow) presented with an absence of an ipsilateral SMCV (SMCV-) (long white arrow) on 4-dimensional computed tomographic angiography (4D-CTA) (A). He received thrombectomy 5 hours after stroke onset, but failed to achieve successful reperfusion [modified thrombolysis in cerebral infarction score ( $\mathrm{m}$ TICI) score $=2 \mathrm{a}$ ] (B). On the 6-hour follow-up, non-contrast CT (NCCT) scan after thrombectomy, parenchymal hematoma (PH), and midline shift were present (C). He then received a decompressive craniectomy and evacuation of the hematoma (D). His NIHSS score and modified Rankin Scale (mRS) score at discharge was 37 and 5, respectively. He died 7 days after stroke onset. Patient 2: A 49-year-old male with an acute left MCAO (white triangle arrow) (baseline NIHSS =22), presented with symmetric contrast filling of the SMCV (SMCV+) (long white arrow) on 4D-CTA (E). He received thrombectomy 5.5 hours after stroke onset and achieved successful reperfusion (mTICI score $=3$ ) $(\mathrm{F})$. There was no significant hemorrhagic transformation on his 24-hour follow-up NCCT (G) or 72-hour follow-up diffusion-weighted imaging (H). His NIHSS score and mRS score at discharge was 16 and 4, respectively. NIHSS, National Institute of Health stroke scale.

these two treatment options was rarely mentioned. In our study, intravenous thrombolysis might have induced clot lysis before thrombectomy or partially lysed the clot to permit some reperfusion before thrombectomy was conducted, resulting in a reduced time of cerebral hypoxia, mitigating irreversible damage $(21,23)$.

As an imaging marker, SMCV- is recognizable, and the identification of SMCV- can be easily completed in the majority of CTP post-processing workstations with excellent inter- and intra-rater agreement. Therefore, its evaluation can be performed routinely before treatment decision-making in centers that have CTP scans. Although thrombectomy is now the primary treatment option for acute LAO, our study suggests that patients with SMCVmay face failure in reperfusion, and a higher risk of hematoma even if reperfusion can be successfully achieved. Therefore, a novel neuroprotective strategy for patients with SMCV- at baseline should be explored in order to provide feasible options in addition to thrombectomy.

This study had several limitations. Firstly, it had a small sample size and was a retrospective study, which created a potential risk of selection bias. Additionally, our subjects might be more severe than other centers in terms of neurological deficits (median NIHSS of 19). Therefore, results should be interpreted with caution. Secondly, as many patients did not receive further CTP, 
we cannot dynamically observe if the presence of SMCVis influenced by reperfusion. Thirdly, the off-label use of tirofiban might have compromised study group homogeneity. Fourthly, we did not provide long-term follow-up data as we focused on observing the short-term changes in the clinical manifestation of acute LAO patients. In acute LAO, patients may suffer rapid deterioration of neurological function, which may even lead to death within several days after stroke onset. Therefore, shortterm outcomes may be more reflective of prognosis than 3 -month long-term outcomes in acute LAO patients. Our future studies will include both short- and long-term outcomes in order to comprehensively judge the predictive value of SMCV - in LAO patients receiving thrombectomy. Lastly, we did not evaluate the effect of other imaging markers on reperfusion after treatment, such as the length of the thrombus. Therefore, further studies are required using a panel of imaging markers to confirm our findings. Future investigations should also have a larger sample size and involve multicenter clinical trials to improve the generalizability of results.

In conclusion, in patients who received thrombectomy, $\mathrm{SMCV}$ - did not directly predict $\mathrm{PH}$, but was a risk factor for reperfusion. Although reperfusion had a protective effect on $\mathrm{PH}, \mathrm{SMCV}$ - patients still had a higher rate of $\mathrm{PH}$ than $\mathrm{SMCV}+$ in patients who achieved successful reperfusion.

\section{Acknowledgments}

Funding: This work was supported by the National Natural Science Foundation of China [81801162 to SZ, 81702462 to CG.L.], National Natural Science the China Postdoctoral Science Foundation [2019M662083 to RT.Z.], Zhejiang Provincial Natural Science Foundation of China [LQ20H180015 to RT.Z.], and General Project Funds from the Health Department of Zhejiang Province [2013KYB024 to $\mathrm{ZW]}$.

\section{Footnote}

Reporting Checklist: The authors have completed the STROBE reporting checklist. Available at http://dx.doi. org/10.21037/atm-20-1154

Data Sharing Statement: Available at http://dx.doi. org/10.21037/atm-20-1154

Peer Review File: Available at http://dx.doi.org/10.21037/ atm-20-1154

Conflicts of Interest: All authors have completed the ICMJE uniform disclosure form (available at http://dx.doi. org/10.21037/atm-20-1154). The authors have no conflicts of interest to declare.

Etbical Statement: The authors are accountable for all aspects of the work in ensuring that questions related to the accuracy or integrity of any part of the work are appropriately investigated and resolved. The study was approved by the institutional ethics committee (NO. KY2017019), and informed consent was obtained from all patients. All clinical investigations were conducted according to the principles expressed in the Declaration of Helsinki (as revised in 2013).

Open Access Statement: This is an Open Access article distributed in accordance with the Creative Commons Attribution-NonCommercial-NoDerivs 4.0 International License (CC BY-NC-ND 4.0), which permits the noncommercial replication and distribution of the article with the strict proviso that no changes or edits are made and the original work is properly cited (including links to both the formal publication through the relevant DOI and the license). See: https://creativecommons.org/licenses/by-nc-nd/4.0/.

\section{References}

1. Larrue V, von Kummer RR, Müller A, et al. Risk factors for severe hemorrhagic transformation in ischemic stroke patients treated with recombinant tissue plasminogen activator: a secondary analysis of the EuropeanAustralasian Acute Stroke Study (ECASS II). Stroke 2001;32:438-41.

2. van Kranendonk KR, Treuniet KM, Boers AMM, et al. Hemorrhagic transformation is associated with poor functional outcome in patients with acute ischemic stroke due to a large vessel occlusion. J Neurointerv Surg 2019;11:464.

3. Lee JS, Hong JM, Kim EJ, et al. Comparison of the Incidence of Parenchymal Hematoma and Poor Outcome in Patients with Carotid Terminus Occlusion Treated with Intra-Arterial Urokinase Alone or with Combined IV rtPA and Intra-Arterial Urokinase. AJNR Am J Neuroradiol 2012;33:175-9.

4. Parthasarathy R, Mahesh K, Rempel JL, et al. Prognostic evaluation based on cortical vein score difference in stroke. 
Stroke 2013;44:2748-54.

5. Cartmell SCD, Ball RL, Kaimal R, et al. Early Cerebral Vein After Endovascular Ischemic Stroke Treatment Predicts Symptomatic Reperfusion Hemorrhage. Stroke 2018;49:1741-6.

6. Zhang S, Lai Y, Ding X, et al. Absent Filling of Ipsilateral Superficial Middle Cerebral Vein Is Associated with Poor Outcome After Reperfusion Therapy. Stroke 2017;48:907-14.

7. Gerber JC, Miaux YJ, von Kummer R. Scoring flow restoration in cerebral angiograms after endovascular revascularization in acute ischemic stroke patients. Neuroradiology 2015;57:227-40.

8. Powers WJ, Rabinstein AA, Ackerson T, et al. 2018 guidelines for the early management of patients with acute ischemic stroke: A guideline for healthcare professionals from the american heart association/american stroke association. Stroke 2018;49:e46-e110.

9. Campbell BCV, Mitchell PJ, Yan B, et al. A multicenter, randomized, controlled study to investigate EXtending the time for Thrombolysis in Emergency Neurological Deficits with Intra-Arterial therapy (EXTEND-IA). Int J Stroke 2014;9:126-32

10. Menon BK, d'Esterre CD, Qazi EM, et al. Multiphase ct angiography: A new tool for the imaging triage of patients with acute ischemic stroke. Radiology 2015;275:510-20.

11. Dargazanli C, Consoli A, Barral M, et al. Impact of modified tici 3 versus modified tici $2 \mathrm{~b}$ reperfusion score to predict good outcome following endovascular therapy. AJNR Am J Neuroradiol 2017;38:90-6.

12. Goyal M, Demchuk AM, Menon BK, et al. Randomized assessment of rapid endovascular treatment of ischemic stroke. N Engl J Med 2015;372:1019-30.

13. Gibson JY, Massingale TW, Graves GR, et al. Relationship of Cranial Midline Shift to Outcome of Very-LowBirth-

Cite this article as: Zhang S, Zhang R, Jin B, Shi Z, Li C, Yu Y, Wang Z. Absent filling of the superficial middle cerebral vein is associated with reperfusion but not parenchymal hematoma in stroke patients undergoing thrombectomy: an observational study. Ann Transl Med 2020;8(21):1410. doi: 10.21037/atm-201154
Weight Infants with Periventricular Hemorrhagic

Infarction. J Neuroimaging 1994;4:212-7.

14. Pranevicius M, Pranevicius O. Cerebral venous steal: blood flow diversion with increased tissue pressure. Neurosurgery 2002;51:1267-73.

15. Pranevicius O, Pranevicius M, Pranevicius H, Liebeskind DS. Transition to collateral flow after arterial occlusion predispose to cerebral venous steal. Stroke 2012;43:575-9.

16. Kulik T, Kusano Y, Aronhime S, et al. Regulation of cerebral vasculature in normal and ischemic brain. Neuropharmacology 2008;5 5:281-8.

17. Zhang JH, Badaut J, Tang J, et al. The vascular neural network-a new paradigm in stroke pathophysiology. Nat Rev Neurol 2012;8:711-6.

18. Fernández-Klett F, Priller J. Diverse functions of pericytes in cerebral blood flow regulation and ischemia. J Cereb Blood Flow Metab 2015;35:883-7.

19. Budohoski KP, Czosnyka M, Kirkpatrick PJ, et al. Clinical relevance of cerebral autoregulation following subarachnoid haemorrhage. Nat Rev Neurol 2013;9:152-63.

20. Linfante I, Cipolla MJ. Improving reperfusion therapies in the era of mechanical thrombectomy. Transl Stroke Res 2016;7:294-302.

21. Maingard J, Shvarts Y, Motyer R, et al. Outcomes of endovascular thrombectomy with and without bridging thrombolysis for acute large vessel occlusion ischaemic stroke. Intern Med J 2019;49:345-51.

22. Phan K, Dmytriw AA, Lloyd D, et al. Direct endovascular thrombectomy and bridging strategies for acute ischemic stroke: a network meta-analysis. J Neurointerv Surg 2019;11:443-9.

23. Barreto $\mathrm{AD}$. Intravenous thrombolytics for ischemic stroke. Neurotherapeutics 2011;8:388-99. 